\title{
Problemas emocionales y de comportamiento en niños con discapacidad intelectual ${ }^{*}$
}

\section{Emotional and behavioural problems in children with intellectual disability}

\author{
Claudia Paola Coronel ${ }^{* *}$ \\ Universidad Nacional de Tucumán, \\ Argentina \\ Recibido: 7 de agosto de 2017 \\ Revisado: 16 de noviembre de 2017 \\ Aceptado: 21 de enero de 2018
}

\section{Resumen}

Estudiar los problemas de comportamiento en los niños resulta complejo y más aún si presentan discapacidad intelectual, ya que se involucran diversos factores etiológicos: contextuales, genéticos, familiares, interaccionales; algunos de ellos pueden ser causa por sí mismos de psicopatología o aumentar el riesgo de psicopatología. Los objetivos de este trabajo fueron identificar problemas emocionales y de comportamiento en niños de 6 y 11 años con discapacidad intelectual de diferentes niveles socioeconómico (NSE) de Tucumán, Argentina. Segundo, analizar comportamientos internalizantes y externalizantes en niños con discapacidad intelectual y analizar si existen diferencias en los problemas emocionales y de comportamiento en función del contexto socioeconómico de los niños. Los hallazgos del presente estudio se relacionan con una mayor problemática comportamental y emocional en niños

Artículo de investigación. Este trabajo fue avalado por la Facultad de Psicología de la Universidad Nacional de Tucumán, Argentina. DOI: https://doi.org/10.15332/s1794-9998.2018.0002.11

Correspondencia: Claudia Paola Coronel, Dra. en Humanidades (Área Psicología). Mg. Psicología Educacional (UNT). Lic. Psicología (UNT). Prof. adjunto de evaluación y diagnóstico psicológico infanto-juvenil. Directora alterna del Departamento de Investigaciones de la Facultad de Psicología de la UNT. Facultad de Psicología, Universidad Nacional de Tucumán. Correo electrónico: paolacoronel.pc@gmail.com. Dirección postal: Frias Silva 1147, Yerba Buena (4107), Tucumán, Argentina. 
con discapacidad intelectual moderada referida a problemas de atención, sociales y de retraimiento. Además se encontró mayor problemática emocional en niños con discapacidad intelectual de niveles socioeconómico bajo de Tucumán.

Palabras clave: problemas emocionales, problemas de comportamiento, niños, discapacidad intelectual.

\section{Abstract}

Studying behavioral problems in children is complex, especially if they have an intellectual disability. The aetiology of such problems is diverse: context, genetics, family and interaction, and some of these factors can be themselves a cause of psychopathology or increase its risk. The aims were: to identify emotional and behavioural problems in 6-to-11-year-old children with intellectual disability from different socioeconomic levels (SEL) from Tucumán, Argentina; to analyse internalizing and externalizing behaviour in children with intellectual disability; and to analyse whether there were differences in emotional and behavioural problems depending on the children's SEL. The findings of this study can be related to greater emotional and behavioural problems in children who have a moderate intellectual disability associated with attention, shyness and social problems. In addition, greater emotional problems were found in children with intellectual disability of low socioeconomic levels from Tucumán.

Keywords: emotional problems, behavioural problems, children, intelectual disability.

\section{Introducción}

La salud mental es un componente fundamental e inseparable de la salud y está relacionada con la calidad de vida, familiar y comunitaria (Mora Antó, Córdoba Andrade, Bedoya Urrego \& Verdugo, 2007). El estudio de las características y prevalencia de los problemas emocionales y de comportamiento en la infancia y adolescencia es un campo de investigación necesario tanto para establecer tratamientos eficaces como para desarrollar programas de prevención.

Los problemas emocionales y de comportamiento en la infancia suponen una preocupación y un reto para padres, educadores y profesionales de la salud ocupando un lugar destacado en las investigaciones sobre trastornos psicopatológicos en niños (Garaigordobil \& Maganto, 2013). Y dentro de estas el diagnóstico y el tratamiento de las enfermedades mentales en niños con Discapacidad Intelectual (DI) se ha convertido en una de las áreas de mayor interés (Márquez-Caravero, Zanabria-Salcedo, Pérez-Barrón, Arciniega-Buenrostro \& Galván-García, 2011).
Según la Organización Panamericana de la Salud (OPS), los trastornos mentales constituyen el $22 \%$ de la carga total de enfermedades en América Latina y el Caribe (Rodríguez, Kohn \& Aguilar-Gaxiola, 2009). Así, diferentes estudios epidemiológicos confirman que entre los 6 y 12 años la consulta psicológica por problemas emocionales y de comportamiento constituyen uno de los motivos de mayor prevalencia con una tasa del $9 \%$ al $22 \%$. En Argentina, un estudio epidemiológico realizado por el Ministerio de Salud de la Nación (2007) en conjunto con las universidades nacionales encontraron un $14,9 \%$ de problemáticas internalizantes y un $14,6 \%$ de problemáticas externalizantes. Este estudio ha permitido establecer que un $4,3 \%$ de niños se encuentran en alta vulnerabilidad y un $10,9 \%$ en la categoría de vulnerabilidad.

Como bien señala Donas Burak (2001) con el desarrollo de sistemas de atención de la salud de los adolescentes se comenzó a emplear el denominado enfoque de riesgo. Así, se utilizaron conceptos tales como factores de riesgo, conductas de riesgo y vulnerabilidad. Se entiende por factores 
de riesgo a aquellas "características detectables en un individuo, familia, grupo o comunidad que 'señalan' una mayor probabilidad de tener o sufrir un daño" (p. 490). Este autor afirma que el nivel de vulnerabilidad es el resultado de la interacción de múltiples factores tanto de protección como de riesgo, de orden biológico, psicosocial y del entorno. La vulnerabilidad es un estado en permanente cambio y debe ser valorada teniendo en cuenta factores protectores generales y específi$\cos$, factores de riesgo generales y específicos y conductas de riesgo existentes. En la discapacidad intelectual, factores protectores generales serían, por ejemplo, contar con adecuados apoyos individualizados $\mathrm{y}$, un factor protector específico, temer amigos con buenos vínculos. En cambio, un factor de riesgo general estaría dado por ser partícipe de violencia intrafamiliar, o la deserción escolar; mientras que puede considerarse como factor especifico de riesgo, a modo de ejemplo, que el niño o adolescente se perciba deprimido o triste (Contini \& Coronel, 2015).

La evaluación psicológica tiene como finalidad conocer y comprender los comportamientos y síntomas que aparecen para determinar si éstos (comportameintos y síntomas) son parte del curso normal del desarrollo o constituyen un patrón psicopatológico (Coronel, 2013). Las alteraciones psicológicas han sido tradicionalmente clasificadas en dos patrones básicos: problemas de comportamiento y de las emociones.

Por un lado, las alteraciones de las emociones o síndromes internalizantes están relacionadas con inestabilidad del estado ánimo, obsesiones, problemas somáticos, nerviosismos, inseguridad, miedos, fobias, tristezas, apatía, disforia, inquietud, tensión, preocupación y culpabilidad (Achenbach \& Rescorla, 2012). Para su diagnóstico se pueden emplear los criterios descritos por la Asociación Americana de Psiquiatría (APA, 2014) en las diversas ediciones del Manual Diagnóstico y Estadístico de los Trastornos Mentales (DSM-V). Están relacionados a problemas afectivos, problemas de ansiedad y a somatizaciones. Las taxonomías empíricas muestran que la sintomatología internalizante no aparece tan claramente delimitada en infancia y adolescencia por lo que Achenbach y colabora- dores $(2001,2012)$ han realizado diferentes análisis factoriales sobre los ítems del Child Behavior Checklist (CBCL) de Achenbach y Rescorla (2001). Los autores de esta prueba han aislado tres dimensiones internalizantes: ansiedad/depresión; aislamiento/depresión y quejas somáticas. La ansiedad y la depresión no se organizaron como síndromes independientes. La configuración de un trastorno mixto ansiedad/depresión en la infancia ha sido identificado recientemente (Achenbach, 2008; Romero Acosta et al., 2010).

Las investigaciones realizadas mediante listados amplios de conducta muestran que los problemas internalizantes son los patrones de alteración psicológica comunes encontrados en infancia, fundamentalmente los temores, miedos y fobias. Es importante destacar que la sintomatología internalizante se relaciona con la vulnerabilidad a desarrollar estrés (López Soler, Alcántara, Fernández, Castro \& López Pina, 2010).

Por otro lado, se consideran alteraciones del comportamiento los síndromes externalizantes, que incluyen comportamientos desajustados como la exteriorización de la agresión, la actuación o descarga impulsiva, agitación psicomotora, desobediencia y comportamiento antisocial (Achenbach \& Edelbrock, 1983). Son más visibles en el contexto familiar y escolar, al manifestarse mediante conductas disruptivas que interfieren en el funcionamiento de los niños en esos ambientes, por lo que son los motivos más frecuentes de derivación o de pedido de consulta. La obtención de este síndrome en diferentes investigaciones y en diversas poblaciones ha demostrado la valides de los prototipos obtenidos empíricamente (López Soler et al., 2010).

Las taxonomías propuestas por Achenbach muestran algunas diferencias por las descriptas en el Manual Estadístico de los Trastornos Mentales y por la Organización Mundial de la Salud: trastornos afectivos, ansiedad, somatizaciones, déficit de atención e hiperactividad, oposicionismo-desafiante y problemas de conducta. A partir del CBCL se puede obtener información tanto de los trastornos descriptos por las clasificaciones internacionales y por los propuestos por Achenbach. 
Este autor propone puntos de corte en población estadounidense (Achenbach \& Rescorla, 2001).

\section{Discapacidad intelectual y salud mental}

Los trastornos mentales y las alteraciones de conducta pueden afectar a todos los niños y adolescentes, tengan o no discapacidad intelectual. La mayor parte de los niños y adolescentes con discapacidad intelectual no desarrollan más problemas de conducta que sus pares sin discapacidad intelectual. Es discutido si los niños con discapacidad intelectual presentan una mayor vulnerabilidad a las enfermedades mentales y también si sus síntomas son enmascarados por el déficit cognitivo. Verdugo Alonso y Gutiérrrez Bermejo (2009) indican que en muchas ocasiones es el contexto el que no favorece el desarrollo de comportamientos apropiados y fomenta repertorios desajustados que llevan a la intervención profesional. Lo que parece evidente es que un elevado número de casos permanecen sin diagnóstico, es decir se encuentran extremas dificultades para identificar los problemas de salud mental, dado que en la mayoría de las ocasiones se mostrarán en forma de conducta desafiante, alteraciones de la conducta, señales físicas y quejas (Novell Alsina, Rueda Quillet \& Salvador-Carulla, 2012).

El concepto vigente de discapacidad intelectual hace referencia a "limitaciones significativas tanto en el funcionamiento intelectual como en la conducta adaptativa tal y como se ha manifestado en habilidades adaptativas conceptuales, sociales y prácticas. Esta discapacidad se origina antes de los 18 años" (Schalock et al, 2011, p. 31). En la actualidad el peso, en relación al diagnóstico, está puesto en la valoración de la conducta adaptativa con la finalidad de reflejar las características sociales de la discapacidad, reducir el exceso de confianza depositado en el coeficiente intelectual y disminuir los falsos positivos (Grossman, 1973; Luckasson et. al. 2004; Schalock, 2011).

El concepto de discapacidad intelectual alude a una evaluación integrada del funcionamiento del sujeto en el momento actual. Esto conlleva una dinámica en su análisis, en contrapartida al enfoque estático asumido en épocas anteriores. Esto es, que se debe comprender a la discapacidad intelectual no como una condición permanente - aquello que se tiene para toda la vida - sino como un estado actual de funcionamiento que debe ser revisado periódicamente y en diversos aspectos de la vida de un individuo.

El déficit cognitivo debe coexistir junto a limitaciones en la conducta adaptativa, puesto que la restricción intelectual, por sí misma, es limitada para diagnosticar discapacidad intelectual. La conducta adaptativa se entiende como "el conjunto de habilidades conceptuales, sociales y prácticas aprendidas por las personas para funcionar en su vida diaria" (Luckasson et al, 2004, p. 73). Las limitaciones en la conducta adaptativa afectan tanto a la vida diaria como a la habilidad para responder a los cambios en la vida y a las demandas ambientales.

Schalock et al (2011) enfatizan que el enfoque multidimensional, base teórica actual para la comprensión de la discapacidad intelectual, describe cómo el funcionamiento y la presencia de discapacidad intelectual implican la interacción dinámica y recíproca entre habilidad intelectual, conducta adaptativa, salud, participación, contexto y apoyos individualizados. Este modelo contempla las etapas: a) Identificar la existencia de discapacidad intelectual; b) Evaluación de la participación, interacción y roles sociales de la persona dentro de los diferentes contextos: familia, escuela, comunidad como factores que restrinjan o faciliten el bienestar personal y c) Consideraciones del estado de salud, incluyendo salud física, mental y factores etiológicos relevantes. En este trabajo se hará referencia dentro del modelo multidimensional a la salud mental de los niños con discapacidad intelectual.

Una de las preocupaciones entre los profesionales que trabajan en el campo de la discapacidad intelectual es el reconocimiento que presentan estos individuos para reconocer los problemas físicos y psíquicos, en comunicar los síntomas y sentimientos. Los niños con discapacidad intelectual están sometidos a diferentes tipos de estrés 
ambiental. Así, niños con dificultades en la comunicación verbal que conviven en grupos, se les exige determinados rendimientos para lograr un mejor ajuste a su medio, pueden tener como resultado comportamientos agresivos como una manera de comunicar sus sentimientos o de asumir algún control de la situación (Novel Alsina et al., 2012). Como es sabido, la manifestación clínica de un trastorno, su gravedad y la afectación sobre el funcionamiento es el resultado de la interacción de múltiples factores y mecanismos.

\section{Prevalencia de los trastornos psicológicos en la Discapacidad Intelectual. Factores de riesgo}

Eaton y Menoslacino (1982) consideran que los sujetos con discapacidad intelectual son más proclives a desarrollar problemas graves de conducta o enfermedades mentales que aquellos que no presentan discapacidad intelectual, debido a sus dificultades para procesar la información, problemas orgánicos, dificultades sensoriales o factores culturales y familiares.

Los posibles factores que influyen en la presencia de problemas de comportamiento (Verdugo Alonso \& Gutiérrez Bermejo, 2009) son:

- Las lesiones del sistema nervioso central y su pobre desarrollo contribuyen a que aumente la probabilidad de comportarse impulsivamente bajo condiciones externas de provocación.

- La presencia de otros trastornos asociados con la discapacidad intelectual, como ser la epilepsia, desórdenes sensoriales, dificultades motrices, parálisis cerebral, trastornos del lenguaje, aumentan las dificultades.

- Las limitaciones en habilidades cognitivas disminuyen la probabilidad de que los niños con discapacidad intelectual puedan superar, de forma asertiva, las fuentes de estrés.

- Determinadas formas de vida que influyen de un modo desajustado en la probabilidad de presentar dificultades comportamentales o emocionales.
La presencia de unos $u$ otros tipos de problemas de comportamiento o emocionales está relacionada con el nivel de discapacidad del sujeto. Es importante subrayar que, a mayores necesidades de apoyo, mayor es la presencia de problemas de comportamiento. Los niños y adolescentes con sistemas de apoyo amplio suelen presentar más problemas de comportamiento de mayor gravedad, como ser las autolesiones, estereotipias o agresiones. Asimismo, los niños con menos necesidades de apoyo pueden presentar las mismas problemáticas emocionales o comportamentales que sus iguales en edad y sin discapacidad intelectual.

Investigaciones realizadas con niños sin discapacidad intelectual y con problemas de comportamiento demuestran que a medida que el desarrollo avanza estos problemas disminuyen o desaparecen (Mangrulkar, Whitman \& Posner, 2001). Mientras que en los niños con discapacidad intelectual suelen permanecer en estadios posteriores del desarrollo. Así el llanto y la agresividad son, en un determinado periodo del desarrollo, formas de comunicación. A medida que el niño crece el lenguaje sustituye a otros comportamientos. Es decir que los niños tienden a reducir o eliminar los problemas de comportamiento tales como gritos, llantos o agresiones, cuando logran una forma de comunicación más competente a través del lenguaje verbal. Verdugo Alonso y Gutiérrez Bermejo (2009) señalan que aquellos niños que no disponen de niveles de lenguaje como el discurso y una adecuada expresión narrativa utilizan formas más primitivas de comunicación, tales como la heteroagresividad, autolesiones y miedos. En este sentido, Etxebarria, Apodaca, Eceiza, Fuentes y Ortiz (2003) en un estudio realizado con 257 niños y niñas de 7 a 9 años encontraron que la conducta agresiva aumentaba en los varones y disminuía en las niñas.

Por lo anterior,los objetivos para este trabajo fueron: 1) identificar problemas emocionales $y$ de comportamiento en niños de 6 y 11 años con discapacidad intelectual de diferentes niveles socioeconómico (NSE) de la provincia de Tucumán, Argentina; 2) analizar problemáticas en las dimensiones internalizantes y externalizantes en niños con discapacidad intelectual y 3 ) analizar si 
existen diferencias en los problemas emocionales y de comportamiento en función del contexto socioeconómico al que pertenecen los niños.

\section{Método}

\section{Participantes}

Participaron 41 niños con discapacidad intelectual entre 6 y 11 años ( 25 varones, $61 \%$; 16 mujeres $39 \%$ ), asistentes a escuelas comunes, públicas y privadas de Tucumán (Argentina). Los niños participantes y sus familias fueron agrupados según su nivel socioeconómico (NSE alto: 26,8 \%, NSE medio: $31,7 \%$ y NSE bajo: $41,5 \%$ ). El $7 \%$ de los niños fue diagnosticado discapacidad intelectual leve. Constituye una submuestra de una investigación más amplia dirigida a estudiar las competencias socio-personales y los problemas de comportamiento en niños con discapacidad intelectual de Tucumán.

\section{Instrumentos}

\section{Child Behavior Checklist (CBCL, Achenbach \& Rescorla, 2001).}

Es un instrumento estandarizado para evaluar la presencia de psicopatología en el niño en los últimos 6 meses. También registra información demográfica, competencias sociales, posibles enfermedades o discapacidades, rendimiento académico, preocupaciones hacia el niño y aspectos positivos de éste. El instrumento está compuesto por 113 ítems con tres alternativas de respuestas $(0=$ no es cierto, 1 = en cierta manera, algunas veces, 2 = muy cierto o cierto a menudo) que los padres responden sobre posibles problemas de conducta que pueden presentar los niños y adolescentes entre 6 y 18 años de edad. Permite obtener puntuaciones en 8 escalas de banda estrecha también llamadas síndromes empíricos: Aislamiento, Ansiedad/Depresión, Quejas somáticas, Problemas sociales, Problemas de pensamiento, Problemas de atención, Comportamiento de romper normas y Conducta agresiva. También permite obtener puntuaciones en 2 escalas de banda ancha: Problemas internalizantes y Problemas externalizantes, además de una puntuación total.

Un estudio realizado en población argentina (Samaniego, 2004) muestra en sus análisis multivariados que se tienen valores elevados de confiabilidad $(, 91)$ lo que indica que este instrumento puede ser usado en investigación epidemiológica en Argentina, como instrumento de screening y en la clínica profesional.

\section{Encuesta sociodemográfica}

La encuesta sociodemográfica fue administrada para identificar el nivel socioeconómico en el grupo objeto de estudio. Se toman como indicadores el nivel de ocupación y educación del jefe del hogar. El nivel socioeconómico se determinó por la combinación de ocupación y educación (Cámara de Empresas de Investigación Social y de Mercado y Asociación Argentina de Marketing, 2006). La variable género fue evaluada como un ítem de la encuesta, a la que los padres responden indicando si su hijo es varón o mujer.

Como único criterio de exclusión del estudio se tuvo en cuenta si el protocolo estaba incompleto o dudoso.

\section{Diseño y procedimiento}

Se trató de un estudio descriptivo, no experimental, de tipo transversal (Hernández Sampieri, Fernández Collado \& Baptista Lucio, 2010). Para la selección de los participantes se realizó una entrevista con el equipo multidisciplinario (pedagogos, psicólogos, fonoaudiólogos y psicomotricistas) que atendían a los niños. De allí, se seleccionaron los casos que cumplían los requisitos para el estudio, niños entre 6 a 11 años de edad con discapacidad intelectual. Los padres participaron de modo voluntario, previo consentimiento informado. La recolección de datos se realizó en las instituciones terapéuticas, con una administración individual de 40 minutos aproximadamente. Para esta presentación se han considerado los niños con discapacidad intelectual leve y moderada. Para 
el diagnóstico de los problemas internalizantes y externalizantes, el criterio empleado fueron las puntuaciones correspondientes al percentil $98 \mathrm{y}$ a la puntuación media más una desviación típica de la muestra en cada una de las escalas del CBCL. Para los niveles socioeconómicos se analizaron indicadores de nivel educativo y ocupación del principal sostén del hogar como la posesión de bienes y servicios. Los datos fueron procesados con el programa SPSS 20.0.

\section{Resultados}

El primer objetivo de este estudio fue identificar problemas de comportamiento y emocionales en los niños de 6 a 11 años, con discapacidad intelectual, de diferentes niveles socioeconómicos de la provincia de Tucumán, Argentina. Un análisis exploratorio determinó que las variables depen- dientes tales como, los problemas sociales, de pensamiento, de atención, de ruptura de reglas, de ansiedad y depresión, retraimiento y depresión, problemas externalizantes e internalizantes presentaban una distribución normal por lo que se decidió emplear pruebas paramétricas.

Se encontró que, según la percepción parental, los niños con discapacidad intelectual moderada presentaban más quejas somáticas $(\mathrm{p}=, 033)$ comparados con sus pares con discapacidad intelectual leve. Si bien no se observó otra diferencia estadística significativa, un análisis de tendencias mostró que los niños con discapacidad intelectual moderada presentaban mayor recurrencia de problemas sociales, de pensamiento y de retraimiento-depresión. En tanto sus pares con discapacidad intelectual leve referían más problemas agresivos (Tabla 1).

Tabla 1.

Comparaciones de media de las dimensiones del CBCL según discapacidad intelectual leve y moderada. Prueba t de Student.

\begin{tabular}{lcccccc}
\hline \multirow{2}{*}{$\begin{array}{c}\text { Dimensiones } \\
\text { CBCL }\end{array}$} & $\begin{array}{c}\text { Discapacidad intelectual } \\
\text { leve }(\mathrm{N}: \text { 31) }\end{array}$ & $\begin{array}{c}\text { Discapacidad intelectual } \\
\text { moderada }(\mathbf{N}: \text { 10) }\end{array}$ & & \\
\cline { 2 - 7 } & $M$ & $D E$ & $M$ & $D E$ & $t$ & $p$ \\
\hline Problemas sociales & 4,64 & 2,99 & 6,7 & 3,09 & $-1,84$ &, 086 \\
\hline Problemas de pensamiento & 1.9 & 2,03 & 3 & 3,43 &,- 95 &, 359 \\
\hline Problemas de atención & 7 & 4,1 & 7,6 & 4,16 &,- 397 &, 697 \\
\hline Rompe reglas & 3,12 & 3,33 & 4,1 & 2,42 &,- 998 &, 330 \\
\hline Agresivos & 5,77 & 5,13 & 5,4 & 2,27 &, 320 &, 751 \\
\hline Depresión & 4,03 & 3,44 & 5,6 & 3,4 & $-1,26$ &, 226 \\
\hline Quejas somáticas & 1,67 & 2,45 & 3,6 & 2,22 & $-2,31$ &, 033 \\
\hline Retraimiento depresión & 3,16 & 3,41 & 5,9 & 4,74 & $-1,68$ &, 117 \\
\hline $\mathrm{p}<, 05$ & & & & & & \\
\hline
\end{tabular}

Fuente: elaboración propia. 
El segundo objetivo del trabajo fue analizar problemáticas en las dimensiones internalizantes y externalizantes en niños con discapacidad intelectual leve y moderada. Los resultados arrojaron mayor presencia de problemas internalizantes en los niños con discapacidad intelectual moderada comprados con sus pares con discapacidad intelectual leve (Tabla 2).

Tabla 2.

Comparaciones entre tipos de discapacidad intelectual según dimensiones internalizantes y externalizantes. CBCL.

\begin{tabular}{lcccccc}
\hline $\begin{array}{c}\text { Variables } \\
\text { Dependientes }\end{array}$ & \multicolumn{1}{c}{$\begin{array}{c}\text { Discapacidad intelectual } \\
\text { leve }(N: \text { 31) }\end{array}$} & $\begin{array}{c}\text { Discapacidad intelectual } \\
\text { moderada }(N: \text { 10) }\end{array}$ & & \\
\hline Problemas internalizantes & 8,87 & 7,38 & 15,1 & 6,69 & $-2,49$ &, 023 \\
\hline Problemas externalizantes & 8,9 & 7,04 & 9,5 & 4,27 & $-3,22$ &, 750 \\
\hline $\mathrm{p}<, 05$ & & & & & & \\
\hline
\end{tabular}

Fuente: elaboración propia.

La prevalencia de las diferentes escalas del CBCL teniendo como criterio diagnóstico la puntuación correspondiente al percentil 98 del $\mathrm{CBCL}$, fue de $13 \%$ problemas sociales; $11 \%$ problemas de pensamiento; $15 \%$ problemas de atención; $13 \%$ problemas de romper reglas; $26 \%$ problemas agresivos; $14 \%$ problemas de ansiedad / depresión; $11 \%$ quejas somáticas; $15 \%$ retraimiento / depresión; 30 \% problemas internalizantes y $31 \%$ problemas externalizantes.

Estos porcentajes disminuyeron al calcular la prevalencia a partir de las medias más una desviación típica de la muestra de niños con discapacidad intelectual: 2,4 \% problemas sociales; 2,4\% problemas de pensamiento; 4,9\% problemas de atención; 2,4 \% problemas de romper reglas; 2,4\% problemas agresivos; $2,4 \%$ problemas de ansiedad / depresión; 2,4 \% quejas somáticas; 4,9\% retraimiento / depresión; 4,9\% problemas internalizantes y $2,4 \%$ problemas externalizantes.

La puntuación de corte de la muestra de niños con discapacidad intelectual ( $X+1 D S)$, fue la que se tuvo en cuenta como criterio para estimar las diferencias entre DI leve y moderada (Tabla 1 y 2).

Posteriormente, se analizó la relación del contexto socioeconómico en los problemas emocionales y de comportamiento de niños con discapacidad intelectual. Si bien no se encontraron diferencias estadísticas entre los grupos, se observó que los niños con discapacidad intelectual pertenecientes al nivel socioeconómico bajo referían mayor frecuencia de problemas de pensamiento respecto a sus pares de otros contextos socioeconómicos (Tabla 3). 
Tabla 3.

Comparaciones entre niveles de socioeconómico (NSE) según escalas CBCL. Análisis univariados.

\begin{tabular}{|c|c|c|c|c|c|c|c|c|}
\hline \multirow[t]{2}{*}{ Variables Dependientes } & \multicolumn{2}{|c|}{ NSE bajo (N: 17) } & \multicolumn{2}{|c|}{ NSE medio (N: 13) } & \multicolumn{2}{|c|}{ NSE alto (N: 11) } & \multirow[b]{2}{*}{$F$} & \multirow[b]{2}{*}{$p$} \\
\hline & M & $D E$ & M & $D E$ & M & $D E$ & & \\
\hline Problemas sociales & 5,29 & 3,7 & 4,76 & 2,65 & 5,36 & 2,83 & ,135 & ,874 \\
\hline Problemas de pensamiento & 3,17 & 2,94 & 1,53 & 1,94 & 1,36 & 1,62 & 2,67 & 082 \\
\hline Problemas de atención & 7,35 & 4,6 & 6 & 3,91 & 8,18 & 3,34 & 885 & 421 \\
\hline Rompe reglas & 3,7 & 3,34 & 2,69 & 2,46 & 3,63 & 3,64 & ,428 & ,665 \\
\hline Agresivos & 5,47 & 3,41 & 5,76 & 6,48 & 5,9 & 3,83 & ,032 & ,968 \\
\hline Depresión & 5,058 & 2,9 & 3,92 & 3,7 & 4 & 4,09 & ,491 & ,616 \\
\hline Quejas somáticas & 2,94 & 3,24 & 1 & 1,47 & 2,27 & 1,73 & 2,36 & , 108 \\
\hline Retraimiento depresión & 3,7 & 2,92 & 3,07 & 2,92 & 4,9 & 5,14 & 659 &, 523 \\
\hline Problemas internalizantes & 11,7 & 7,96 & 8 & 6,01 & 11,18 & 8,79 & 945, & 398, \\
\hline Problemas externalizantes & 9,17 & 6,24 & 8,46 & 7,55 & 9,54 & 5,82 & ,086 & ,918 \\
\hline
\end{tabular}

Fuente: elaboración propia.

\section{Discusión}

Generalmente son los padres quienes suelen detectar problemas emocionales o de comportamiento en los niños. Los estudios epidemiológicos confirman que entre los 6 y los 12 años los problemas de comportamiento son los motivos de mayor prevalencia y de consulta a profesionales de la salud mental. Los resultados encontrados dan cuenta que los niños con discapacidad intelectual moderada presentan problemas de atención, sociales y retraimiento. Presentan timidez y retraimiento, Son niños poco asertivos y con déficits en las relaciones de interacción social. Los problemas de atención dan cuenta de la dificultad para concentrarse y prestar atención, tanto en horas de clase como fuera de ella. Esto es que se dispersan fácilmente sin poder terminar lo que han iniciado.

Investigaciones con respecto a los problemas de retraimiento son relativamente escasos, sobre todo en población infantil con discapacidad intelectual. Los resultados encontrados en este estudio indican una mayor presencia de estos problemas en niños con una discapacidad intelectual moderada que sus pares con discapacidad intelectual leve. En general, este tipo de comportamiento suelen ser menos visibles que los problemas de conducta como ser los de agresividad, por lo tanto, son menos identificados como problemática a tener en cuenta desde los adultos. Los niños que presentan retraimiento suelen ser impopulares, presentan dificultades en las relaciones sociales y presentan dificultades en el establecimiento de las relaciones sociales.

Es así comoRieffe, Meerum Terwogt, y Bosch (2004) sugieren que los estados de ánimo negativos son los mediatizadores entre la identificación de las emociones y las quejas somáticas. Esto sugiere que una incapacidad percibida para identificar las emociones propias, así como una decreciente tendencia a reconocer las emociones, impide a la persona afrontar de forma adaptativa la situación emocional. Los estados de ánimo negativos contribuyen a la aparición de más quejas somáticas. 
Con respecto al segundo objetivo, identificar presencia de problemas internalizantes y externalizantes. Los resultados encontraron en este estudio dan cuenta de mayor presencia de problemas internalizantes en los niños con discapacidad intelectual moderada. Estos resultados correlacionan con los hallados por Trianes, Blanca, García y Sánchez (2003) quienes encontraron mayor ansiedad y problemas internalizantes en niños con discapacidad intelectual integrados en aulas comunes de la ciudad de Málaga, España.

Los problemas internalizantes se caracterizan por ansiedad, depresión, aislamiento y quejas somáticas, son comportamientos con contenido emocional y expresan un modo desadaptativo de resolver conflictos, en el sentido de que la expresión de los mismos es de orden interno (inhibición, alteraciones en el pensamiento, en el desarrollo y en la autonomía, retraimiento, depresión y ansiedad (Achenbach, 2008).

Los resultados encontrados en el presente estudio se relacionan con los de Eaton y Menoslacino (1982) quienes encontraron que los sujetos con discapacidad intelectual son más proclives a desarrollar problemas graves de conducta o enfermedades mentales que aquellos que no presentan discapacidad intelectual, debido a sus dificultades para procesar la información. La presencia de este tipo de problemas están relacionados con el nivel de discapacidad del sujeto (Mangrulkar, Whitman \& Posner, 2001).

Por último, se ha estudiado la relación entre problemas de comportamiento y nivel socioeconómico. Si bien no se encontraron diferencias estadísticas entre los grupos, se observó que los niños con discapacidad intelectual pertenecientes al nivel socioeconómico bajo referían mayor frecuencia de problemas de pensamiento respecto a sus pares de otros contextos socioeconómicos, identificándose problemas referidos a obsesiones, dificultades en el sueño, comportamientos extraños.

El estudio de los problemas de comportamiento en los niños resulta complejo, puesto que involucra múltiples factores etiológicos, como los contextuales, los genéticos, el ambiente familiar, la interacción con los padres y la vivencia de experiencias traumáticas; algunos de ellos pueden ser causa por sí mismos de psicopatología o aumentar el riesgo de presencia de sintomatología psicopatológica (Valencia García \& Andrade Palos, 2004). El niño es un ser biopsicosocial en constante interacción con el ambiente, tenga o no discapacidad intelectual (Bronfenbrenner, 2005). A su vez, la influencia del contexto social sobre la familia configura modos de interrelaciones específicos entre sus miembros (Heredia \& Ancona, 2014).

\section{Referencias}

Achenbach T. \&, Rescorla L. (2001). Manual for the ASEBA School-Age Forms \& Profiles. Burlington, VT: University of Vermont, Research Center for Children,Youth, \& Families.

Achenbach, T. \& Edelbrock, C. (1993). Diagnóstico, taxonomía y evaluación. En: T. Ollendick. \& M. Hersen (1993). Psicopatología infantil. Barcelona: Martínez Roca.

Achenbach, T. \& Rescorla, L. (2012). International epidemiology of child and adolescent psychopathology 1: diagnoses, dimensions, and conceptual issues. Journal of the American Academy of Child and Adolescent Psychiatry, 51, 1261-1272.

Achenbach, T. (2008). Assessment, diagnostic, nosology and taxonomy of child and adolescent psychopathology. Handbook of clinical psychology, NJ: John Wiley.

American Psychiatric Association, APA (2014). Manual diagnóstico y Estadístico de los trastornos mentales, DSM IV-TR. Barcelona: Masson.

Bronfenbrenner, U. (2005). Making human beings human. Bioecological Perspectives on Human Development. Thousand Oaks: Sage.

Cámara de Empresas de Investigación Social y de Mercado y Asociación Argentina de Marketing (2006). Nivel socioeconómico social. Buenos Aires: Asociación Argentina de Marketing. 
Contini, N. \& Coronel, P. (2015). Las habilidades sociales en la infancia y adolescencia. Conceptos y marco teórico. En N. Contini (Comp.), Agresividad en los adolescentes hoy. Las habilidades sociales como clave para su abordaje (pp. 13-59). Tucumán: EDUNT.

Coronel, P. (2013). La evaluación psicológica como intervención preventiva en el campo de las habilidades cognitivas en la infancia. (Trabajo monográfico inédito). Cátedra Teoría y Técnicas de Exploración Psicológica (Niños). Universidad Nacional de Tucumán.

Donas Burak, S. (2001). Protección, riesgo y vulnerabilidad: Sus posibles aplicaciones en la promoción, prevención, tratamiento y rehabilitación de la salud integral de los adolescentes y las adolescentes. En S. Donas Burak. (Edt.) Adolescencia y juventud en América Latina (489-499). Cartago: Libro Universitario Regional.

Eaton, L. \& Menoslacino, F. (1982). Psychiatric disorders in the mentally retarded: gTypes, problems and challenges. American Journal of Psychiatry, 139, 1297-1303.

Etxebarria, Apodaca, Eceiza, Fuentes \& Ortiz (2003). Diferencia de género en emociones y en conducta social, en la edad escolar. Infancia y Aprendizaje: Diario de Estudios de la Educación y el Desarrollo, 26, 2, 147-161.

Garaigordobil, M., \& Maganto, M. (2013). Problemas emocionales y de conducta en la infancia: un instrumento de identificación y prevención temprana. Padres y maestros, 351, 34-39.

Grossman, H. (1973). A manual on terminology and classification in mental retardation. Washington, DC: American Association on Mental Deficiency.

Heredia y Ancona, M. (2014). Influencia del contexto familiar y social en el desarrollo del niño y sus alteraciones. En E. Lucio GómezMaqueo \& M. Heredia y Ancona (Eds.), Psicopatología. Riesgo y tratamiento de los problemas infantiles (pp. 27-48). México: El Manual Moderno.

Hernández Sampieri, Fernández Collado \& Baptista Lucio (2010). Metodología de la Investigación ( $5^{\circ}$ edición). México: Mc Graw Hill

López Soler, C., Alcántara, M., Fernández, V., Castro, M., \& López Pina, J. (2010). Características y prevalencia de los problemas de ansiedad, depresión y quejas somáticas en una muestra clínica infantil de 8 a 12 años, mediante el CBCL. Child Behavior Checklist. Anales de Psicología, 26, 2, 325-334.

Luckasson, R., Bortwick-Duffy, S., Buntix, W., Coulter, D., Craig, E., ... \& Tassé, M. (2002). Mental Retardation: Definition, classification, and systems of supports. Washington, DC: American Association on Mental Retardation].

Mangrulkar, L., Whitman, C., \& Posner, M. (2001). Enfoques de habilidades para la vida para un desarrollo saludable de niños y adolescentes. Organización Panamericana de la Salud.

Márquez-Caravero, M., Zanabria-Salcedo, M., Pérez-Barrón, E., Arciniega-Buenrostro, L., \& Galván-García, C. (2011). Epidemiología y manejo integral de la Discapacidad Intelectual. Salud Mental, 34, 443-449.

Ministerio de Salud de la Nación y Unidades Académicas de Psicología de Universidades Nacionales. (2007). Problemáticas de Salud Mental en la Infancia. Proyecto de investigación. Informe final. Disponible en www.youblisher. com/files/publications/12/68360/pdf.pdf

Mora Antó, A., Córdoba Andrade, L., Bedoya Urrego, A. \& Verdugo, M. (2007). Características de la calidad de vida de las familias con un adulto con discapacidad intelectual (DI)/RM en la ciudad de Cali, Colombia. Revista Diversitas. Perspectivas en Psicología, 3(1), 37-54.

Novell Alsina, R., Rueda Quitllet, P., Salvador-Carulla, L., \& Forgas Farre, E. (2003). Salud Mental y alteraciones de la conducta en personas con discapacidad intelectual. Guía práctica 
para técnicos y educadores. FEAPS. Disponible en http://hdl.handle.net/11181/3215.

Rieffe, C., Meerum Terwogt, M., \& Bosch, J. (2004). Emotion understanding in children with frequent somatic complaints. European Journal of Developmental Psychology, 1, 31-47.

Rodríguez, J., Kohn, R. \& Aguilar-Gaxiola, S. (2009). Epidemiología de los trastornos mentales en América Latina y el Caribe. Washington, DC: Organización Panamericana de la Salud.

Romero Acosta, K., Canals, J., Hernández-Martínez, C., Jané Balladriga, M., Viñas, F. \& Domènech-Llaberia, E. (2010). Comorbilidad entre los factores de ansiedad SCARED y la sintomatología depresiva en niños de 8-12 años. Psicothema, 22(4), 613-618.

Samaniego, C. (2004). El Child Behavior Checklist: su estandarización en población urbana argentina. Revista de Psicología UCA.
Schalock, R., Borthwick-Duffy, S., Bradley, V., Buntinx, W., Coulter, D., Craig, E... \& Yeager M., (2011). Discapacidad Intelectual. Definición, Clasificación y Sistemas de apoyo. Asociación Americana de Discapacidades Intelectuales y del Desarrollo. Madrid: Alianza. Undécima edición.

Trianes, M., Blanca, M., García, J., \& Sánchez, A. (2003). Competencia social en alumnos con necesidades educativas especiales: nivel de inteligencia, edad y género. Revista de Psicología general y aplicada, 56(3), 325-338.

Valencia García, M. \& Andrade Palos, P. (2004). Validación del Youth Self Report para problemas de conducta en niños mexicanos. International Journal of Clinical and Health Psychology, 5(3), 499-520.

Verdugo Alonso, M., \& Gutiérrez Bermejo, B. (2009). Discapacidad intelectual. Adaptación social y problemas de comportamiento. Madrid: Pirámide. 